the

音 abdus salam

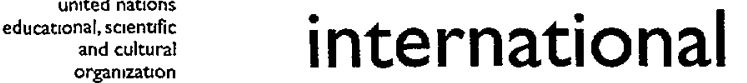
centre for theoretical physics

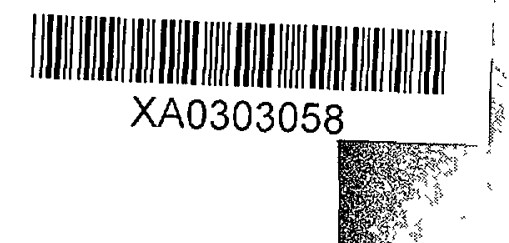

\author{
G. Cassier \\ H. Mahzouli \\ and \\ E.H. Zerouali
}


United Nations Educational Scientific and Cultural Organization and International Atomic Energy Agency

THE ABDUS SALAM INTERNATIONAL CENTRE FOR THEORETICAL PHYSICS

\title{
GENERALIZED TOEPLITZ OPERATORS AND CYCLIC VECTORS
}

\author{
G. Cassier ${ }^{1}$ \\ Institut Girard Desargues, Université Claude Bernard Lyon I, \\ Villeurbanne Cedex, France \\ and
}

The Abdus Salam International Centre for Theoretical Physics, Trieste, Italy,

H. Mahzouli

Département de Mathématiques et Informatiques, Faculté des Sciences de Rabat, B.P. 1014, Rabat, Morocco

and

E.H. Zerouali ${ }^{2}$

Département de Mathématiques et Informatiques, Faculté des Sciences de Rabat, B.P. 1014, Rabat, Morocco

and

The Abdus Salam International Centre for Theoretical Physics, Trieste, Italy.

MIRAMARE - TRIESTE

April 2003

\footnotetext{
${ }^{1}$ cassier@desargues univ-lyon1.fr
}

${ }^{2}$ zerouali@ictp.trieste it 


\begin{abstract}
We give in this paper some asymptotic Von Neumann inequalities for power bounded operators in the class $C_{\rho} \cap C_{1}$, and some spacial von Neumann inequalities associated with non zero elements of the point spectrum, when it is non void, of generalized Toeplitz operators. Introducing perturbed kernel, we consider classes $C_{R}$ which extend the classical classes $C_{\rho}$. We give results about absolute continuity with respect to the Haar measure for operators in class $C_{R} \cap C_{1}$. This allows us to give new results on cyclic vectors for such operators and provides invariant subspaces for their powers. Relationships between cyclic vectors for $T$ and $T^{*}$ involving generalized Toeplitz operators are given and the commutativity of $\{T\}^{\prime}$, the commutant of $T$ is discussed.
\end{abstract}

\title{
1 Introduction
}

Throughout of this paper, we denote $H^{2}$ the Hardy space of analytic functions on the unit disc $D, S$ the unilateral unweighted shift on $H^{2}$ and $S^{*}$ its adjoint operator. Toeplitz operators are defined as solutions of the operator equation $S^{*} X S=X$. They have been intensively treated during the last years and turn out to be a rich source of examples and counter examples in operator theory. Generalized Toeplitz operators were introduced as follows. Let $H$ be a complex Hilbert space and $T \in B(H)$, the algebra of all bounded operators on $H$. The set of $T$-Toeplitz operators, $C(T)$ is defined to contain operators satisfying the operator equation $T^{*} \operatorname{discr}(T)^{2} X$, where $r(T)$ is the spectral radius of $T$. We denote $C_{+}(T)$ as the set positive $T$-Toeplitz operators.

Recall first the following known result. Let $\rho>0$, and $T \in B(H)$ whose spectrum is included in the closed unit disc is in $C_{\rho}$ if and only if $K_{r, t}^{\rho}(T) \geq 0$ for every $\left.\left.(r, t) \in\right] 0,1\right] \times \mathbf{R}$. This leads to the next definition.

Definition 1 Let $R$ be a polynomial and let $T$ be an operator acting on a Hilbert space, we say that $T$ is in class $C_{R}$ if and only if

$$
\sigma(T) \subset \mathbf{D} \quad \text { and } \quad K_{r, t}^{R}(T)=K_{r, t}(T)+R\left(r e^{-\imath t} T\right)+R\left(r e^{\imath t} T\right)^{*}-I \geq 0 .
$$

Remark. When $R=\rho>0$ the class $C_{R}$ is exactly the usual class $C_{\rho}$.

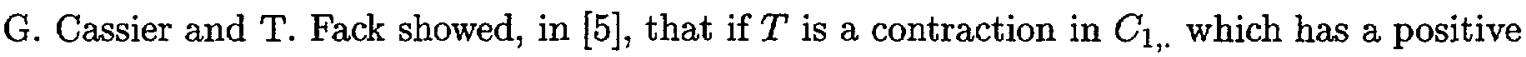
generalized Toeplitz operator with eigenvalues, then $T$ has a non zero noncyclic element, that is a non zero vector $y \in H$ such that $\operatorname{span}\left\{T^{n} y: n \geq 0\right\} \neq H$. In particular $T$ has a non trivial invariant subspace. To this aim, they provided some spacial Von Neumann inequalities for $T$.

In this paper we study the structure of cyclic vectors for powers of power bounded operators in the class $C_{R} \cap C_{1}$. See section 2 for definitions. 
In Section 2, basic background and some known results are given. We recall the notion of almost convergence and Banach limits. We also relate these notions to generalized Toeplitz operators and define the asymptotic kernel for power bounded operators.

In Section 3, we establish the Von Neumann spacial inequality for operators in the class $C_{\rho} \cap C_{1, .}$. We refine this inequality in the case where $T$ has a generalized Toeplitz operator with non void point spectrum.

We investigate in Section 4 the problem of cyclic vectors for power bounded operators in $C_{R} \cap C_{1, .}$. We show in Theorem 14, that completely non unitary operators in $C_{R} \cap C_{1, \text {, are }}$ intertwined with the unilateral shift on some Hilbert space in a satisfactory way. This leads to an extension of a result of Nagy Foias [8], "there exists $N$ such that $T^{n}$ has no cyclic vector for $n \geq N$ ". Other results from [7] are generalized. The use of generalized Toeplitz operators allows to give more precise results in the general case of power bounded operators. Namely, we link cyclic vectors for $T$ and $T^{*}$ through generalized Toeplitz operators. We finally study the commutativity of the commutant of completely non unitary power bounded operators in the class $C_{1, \text {. }}$.

In section 5 , we provide spacial von Neumann inequalities associated to perturbed kernel. We give explicit computations in the case where $R(T)=\beta T+\bar{\beta} T^{*}+I$. This shed light on the role of the existence of eigenvalues of a generalized Toeplitz vector associated with $T$.

\section{Preliminaries}

\subsection{Generalized Toeplitz Operators}

A complex sequence $\xi \in l^{\infty}(\mathbb{N}, C)$ almost converges to the complex number $c$ if

$$
\lim _{k \rightarrow \infty} \sup _{n \in \mathbb{N}}\left|c-k^{-1} \sum_{j=n}^{n+k-1} \xi(j)\right|=0
$$

The sequence $\xi$ is said to be almost convergent in the strong sense to $c$ if $|\xi-c .1|$ is almost convergent to 0 , where 1 is the constant sequence of value 1 .

A Banach limit $L$ is a positive functional on $l^{\infty}$ satisfying

1. $L\left(\left(u_{n}\right)_{n \geq 0}\right)=1$ for $u_{n} \equiv 1$

2. $L\left(\left(u_{n}\right)_{n \geq 0}\right)=L\left(\left(u_{n+1}\right)_{n \geq 0}\right)$

3. If $\left(u_{n}\right)_{n \geq 0}$ converges, then $L\left(\left(u_{n}\right)_{n \geq 0}\right)=\lim _{n \rightarrow+\infty} u_{n}$.

We denote by $\mathcal{B}$ the set of all Banach limits.

The following classes of operators were considered in [9]

$$
C_{1, .}=\left\{T \in B(H) / \forall x \in H \backslash\{0\}, \mid T^{n} x \| \text { does not converges to zero }\right\}
$$




$$
C_{., 1}=\left\{T \in B(H) / \forall x \in H \backslash\{0\}, \mid\left(T^{*}\right)^{n} x \| \text { does no converges to zero }\right\}
$$

and

$$
C_{1,1}=C_{, 1} \cap C_{1, .}
$$

Let $P W B(H):=\left\{T \in B(H): \sup _{n \geq 0}\left\|T^{n}\right\|<+\infty\right\}$ be the set of power bounded operators. Simple computations permit to show that if $T \in P W B(H)$ is such that $\lim _{n \geq 0}\left\|T^{n}\right\| \neq 0$ then the spectral radius $r(T)$ of $T$ equals 1 . This last affirmation will be assumed to be true for all our operators.

Let $T$ be a power bounded operator and $L$ a Banach limits. Consider the functional

$$
y \mapsto(x \mid y):=L\left(\left(<T^{n} x \mid T^{n} y>\right)_{n \geq 0}\right)
$$

Using the positivity of Banach limits, we see that

$$
|(x \mid y)| \leq M^{2}\|x\|\|y\|,
$$

where $M=\sup _{n \geq 0}\left\|T^{n}\right\|$. In particular (.|.) is continuous and by Reisz representation theorem, there exists a positive bounded operator $X_{L}$ such that $(x \mid y)=\left\langle X_{L} x \mid y\right\rangle$. Following [4] such operators are called canonical generalized Toeplitz operators and their set is denoted $\tau(T)$. Recall that, $\tau(T)$ is reduced to a singleton if and only if the sequence $\left(T^{*}\right)^{n} T^{n}$ is weakly almost convergent. In every finite Von Neumann algebra, the sequence $\left(T^{*}\right)^{n} T^{n}$ is always weakly almost convergent [4]. If $T$ is in the class $C_{\rho}$, then $\left(T^{*}\right)^{n} T^{n}$ converges in the strong topology of operators, ([6]). In particular, in both cases $\tau(T)$ is reduced to a singleton.

We collect in the following proposition some properties of canonical Toeplitz operators, for the proof see [4].

Proposition 2 Let $T$ be a Power Bounded Operator and $X \in$. Then

a. $\tau(T) \subset C_{+}(T)$.

b. $\tau(T)$ contains an injective element if and only if $X$ is injective for any $X \in \tau(T)$.

c. $\left\{x \in H\right.$ such that $\left.T^{n} x \rightarrow 0\right\} \subset \operatorname{ker}(X)$ for any $X \in \tau(T)$. In particular, $T \in C_{1}$, when $\tau(T)$ contains an injective element.

We assume for the following theorem that $T \in C_{1, \text {. }}$ and let $X \in \tau(T)$. The associated mapping (.|.) induces an inner product on $H$. Denote by $\widetilde{H}$ the Hilbert completion of $H$ with respect to the norm $\mid\|$.$\| \| , induced by the inner product (.|.) and denote by \tilde{T}$ the canonical extension of $T$ to $\widetilde{H}$. For every $x \in H$ we have

$$
\begin{aligned}
\|\widetilde{T} x\|^{2} & =(\tilde{T} x \mid \widetilde{T} x)=L\left(\left(<T^{n+1} x \mid T^{n+1} x>\right)_{n \geq 0}\right) \\
& =L\left(\left(<T^{n} x \mid T^{n} x>\right)_{n \geq 0}\right) \\
& =\left.\|x\|\right|^{2} .
\end{aligned}
$$


Since $H$ is a dense subspace of $\widetilde{H}$, we conclude that $\widetilde{T}$ is an isometry.

In the invariant subspace problem, since $\overline{\Im(T)}$ and $\overline{\operatorname{ker}(T)}$, if non trivial, provide invariant subspaces. It is always assumed (and we will do) that $T$ has a dense range. This forces $\tilde{T}$ to be dense range, and so $\widetilde{T}$ becomes a unitary transformation. Let $\mu_{0}$ be the spectral measure on $S^{1}=\left\{e^{\imath t} / t \in \mathbb{R}\right\}$ associated with $\tilde{T}$. Consider for $f \in L^{\infty}\left(S^{1}, \mu_{0}\right)$ the invariant subspace introduced by $\mathrm{B}$. Beauzamy in [1]:

$$
E_{f}=H \cap \operatorname{Ker}(f(\tilde{T}))
$$

In [5], it is shown, when $T$ is a contraction, that $E_{f}=\operatorname{Ker} X_{f}$ for some positive generalized Toeplitz operator $X_{f}$ associated with $T$. Outlining the proof of this result, we generalize as follows

Theorem 3 ([5], theorem 7) Let $T$ be a cyclic power bounded operator acting on Hilbert space $H$ with dense range, assume that $T$ is completely non unitary (c.n.u for short) in the class $C_{1, .}$. For $f \in L^{\infty}\left(S^{1}, \mu_{0}\right)$, let $E_{f}$ be the closed invariant subspace generated by $f$. Then, there exists $X_{f} \in C_{+}(T)$ such that $E_{f}=\operatorname{Ker}\left(X_{f}\right)$.

We end this section by recovering some properties of generalized Toeplitz operators associated with power bounded operators. Denote $p_{M}=: M$ the constant gauge, we have

Proposition 4 Let $T$ be a power bounded operator, $\left\|T^{n}\right\| \leq M$ and let $X_{L} \in C_{+}(T)$ be associated with a Banach limit $L_{p_{M}}$. Then for $X \in C_{+}(T)$ we have

$$
0 \leq X \leq M^{2}\|X\| X_{L}
$$

In particular $\operatorname{ker}(X) \subset \operatorname{ker}\left(X_{L}\right)$ and hence if $X_{L}$ is one to one, then every $X \in C_{+}(T)$ is one to one.

Proof. Let $x \in H$,

$$
|<X x| x>|=|<\left(T^{*}\right)^{d_{i s c n}}|x>|=\left|<X T^{n} x\right| T^{n} x>\mid \leq\|X\|\left\|T^{n} x\right\|^{2}
$$

By using the $p-$ Banach limit we obtain

$$
<X x\left|x>\leq M^{2}\|X\|<X_{L} x\right| x>
$$

\section{$3 \quad$ Asymptotic spacial Von Neumann inequalities for operators in $C_{\rho}$}

\subsection{Asymptotic kernels}

Let $T \in P W B(H)$ be such that $r(T)=1$ and $X \in B(H)$ be an arbitrary operator. The asymptotic kernel associated with $X$ is the operator valued function,

$$
K_{r, t}^{\infty}(T, X)=X\left(I-r e^{-\imath t} T\right)^{-1}+\left(I-r e^{\imath t} T^{*}\right)^{-1} X-X
$$


and the kernel associated with $T$ is

$$
K_{r, t}(T)=K_{r, t}^{\infty}(T, I)=\left(I-r e^{-\imath t} T\right)^{-1}+\left(I-r e^{\imath t} T^{*}\right)^{-1}-I
$$

for $r$ such that $(r, t) \in[0,1] \times[0,2 \pi[$.

These kernels were studied in [5] and applied to obtain asymptotic spacial Von Neumann inequalities which allowed the authors to provide invariant subspaces for some classes of contractions.

The following proposition is inspired from [5] assemble some properties of the asymptotic kernel and the proof runs similarly.

Proposition 5 ([5]) Let $T$ be a power bounded operator, $X \in C_{+}(T)$ and let $K_{r, t}^{\infty}(T, X)$ be the asymptotic kernel associated with $X$ defined on $(r, t) \in[0,1] \times[0,2 \pi[$. Then

1. $K_{r, t}^{\infty}(T, X) \in C_{+}(T)$ and satisfies

$$
K_{r, t}^{\infty}(T, X)=\left(1-r^{2}\right)\left(I-r e^{\imath t} T^{*}\right)^{-1} X\left(I-r e^{-\imath t} T\right)^{-1}
$$

2. For $f$ an analytic function on neighborhood of the unit closed disc, we have

$$
X f(r T)=\int_{0}^{2 \pi} f\left(e^{2 t}\right) K_{r, t}^{\infty}(T, X) \frac{d t}{2 \pi}
$$

and

$$
f\left(r T^{*}\right) X=\int_{0}^{2 \pi} f\left(e^{-2 t}\right) K_{r, t}^{\infty}(T, X) \frac{d t}{2 \pi}
$$

for every $\leq r<0$.

Using Proposition 3, We get

Proposition 6 Let $T$ be a power bounded operator, then for every $X \in C_{+}(T)$ and $(r, t) \in$ $[0,1] \times\left[0,2 \pi\left[\right.\right.$, we have $K_{r, t}^{\infty}(T, X) \in i n C_{+}(T)$ and

$$
K_{r, t}^{\infty}(T, X) \leq\|X\| K_{r, t}^{\infty}\left(T, X_{L}\right)
$$

where $X_{L} \in C_{+}(T)$ is associated with a Banach limit $L$.

Proof.

$$
\begin{aligned}
K_{r, t}^{\infty}(T, X) & =\left(1-r^{2}\right)\left(I-r e^{\imath t} T^{*}\right)^{-1} X\left(I-r e^{-\imath t} T\right)^{-1} \\
& \leq\left(1-r^{2}\right)\|X\|\left(I-r e^{\imath t} T^{*}\right)^{-1} X_{L}\left(I-r e^{-\imath t} T\right)^{-1} \\
& \leq\|X\| K_{r, t}^{\infty}\left(T, X_{L}\right)
\end{aligned}
$$




\subsection{Asymptotic spacial Von Neumann inequalities and point spectrum of generalized Toeplitz operators.}

We fit the generalized von Neumann inequality given in [3] to our situation. The use of asymptotic kernels in the proof was crucial. For $X \in C_{+}(T), r \in[0,1[$ and $x \in H$, denote $d m_{r, x}^{X}(t)=<K_{r, t}^{\infty}(T, X) y \mid y>\frac{d t}{2 \pi}$, then

Proposition 7 Let $T$ be a power bounded operator and $X \in C_{+}(T)$. Then for any pair $(f, g)$ of analytics functions on a neighborhood of the closed unit disc, $x \in H$ and $0 \leq r \leq 1$, we have

$$
\left\|\left[f\left(r T^{*}\right) X+X g(r T)\right] x\right\|^{2} \leq\|X\| \int_{0}^{2 \pi}\left|f\left(e^{-\imath t}\right)+g\left(e^{2 t}\right)\right|^{2} d m_{r, x}^{X}(t) .
$$

We develop in the rest of this section asymptotic spacial Von Neumann inequalities for power bounded operators in $C_{\rho}$.

Proposition 8 Let $T$ be a power bounded operator and $X \in C_{+}(T)$. Suppose that $X$ has a nonzero eigenvalue $\alpha$ and let $y$ be a corresponding normalized eigenvector. Let $P, Q$ be polynomials, then

$$
|<Q(T) P(T) y| y>\mid \leq \frac{1}{\alpha}\|\sqrt{X} P(T) y\|\|\sqrt{X} Q(T) y\|
$$

Proof. Simple computations give

$$
<K_{r, t}^{\infty}(T, X) y\left|y>=\alpha<K_{r, t}(T) y\right| y>
$$

For $(r, t) \in[0,1] \times[0,2 \pi[$ and for all polynomials $P, Q$, we obtain

$$
\begin{aligned}
\mid\langle Q(r T) P(r T) y|y>| & =\left|\int_{0}^{2 \pi} P\left(e^{\imath t}\right) Q\left(e^{\imath t}\right)<K_{r, t}(T) y\right| y>\frac{d t}{2 \pi} \mid \\
& =\frac{1}{\alpha}\left|\int_{0}^{2 \pi} P\left(e^{2 t}\right) Q\left(e^{\imath t}\right) d m_{r, y}^{X}(t)\right|
\end{aligned}
$$

Since $K_{r, t}^{\infty}(T, X)$ is a positive operator, by applying the Cauchy-Schwarz inequality we obtain

$$
|<Q(r T) P(r T) y| y>\left|\leq \frac{1}{\alpha} \int_{0}^{2 \pi}\right| P\left(e^{\imath t}\right) Q\left(e^{\imath t}\right) \mid\left\|\sqrt{K_{r, t}^{\infty}(T, X)} y\right\|\left\|\sqrt{K_{r, t}^{\infty}(T, X)} y\right\| \frac{d t}{2 \pi}
$$

Denote $I$ the left side of the inequality above and apply the Cauchy-Schwarz inequality again

$$
\begin{aligned}
I & \leq \frac{1}{\alpha}\left(\int_{0}^{2 \pi}\left|P\left(e^{2 t}\right)\right|^{2} d m_{r, y}^{X}(t)\right)^{\frac{1}{2}}\left(\int_{0}^{2 \pi}\left|Q\left(e^{i t}\right)\right|^{2} d m_{r, x}^{X}(t)\right)^{\frac{1}{2}} \\
& =\frac{1}{\alpha}\|\sqrt{X} P(r T) y\|\|\sqrt{X} Q(r T) y\| .
\end{aligned}
$$

Equation 2 is then obtained by taking $r \rightarrow 1$. The proposition is proved.

As in [5] we associate with an operator $T$, in $C_{\rho}$, the kernel is given by

$$
K_{r, t}^{\rho}(T)=\left(I-r e^{-\imath t} T\right)^{-1}+\left(I-r e^{\imath t} T^{*}\right)^{-1}+(\rho-2) I=K_{r, t}(T)+(\rho-1) I .
$$

Note that $\rho$-asymptotic kernels allow to reformulate the definition of the class $C_{\rho}$. More precisely an operator $T$ belongs to $C_{\rho}$ if the associated $\rho$-asymptotic kernel $\left(K_{r, t}^{\rho}\right)$ is positive and 
if $\rho$ is minimal in this sense. See [5] for further information.

Let $P_{1}, P_{2}$ be polynomials. Fix $I(y)=\left\|\left(P_{1}\left(r T^{*}\right)+P_{2}(r T)\right) y\right\| \|^{2}$ and set $J(x)=<\left(P_{1}\left(r T^{*}\right)+\right.$ $\left.P_{2}(r T)\right) y \mid x>$ for $x \in H$. The following provides asymptotic spacial Von Neumann inequalities for operators in the class $C_{\rho}$

Proposition 9 Under the assumptions above, we have

$$
\begin{aligned}
I(y) & \leq \frac{1}{\alpha} \int_{0}^{2 \pi}\left|(1-\rho)\left(P_{1}(0)+P_{2}(0)\right)+P_{1}\left(e^{-i t}\right)+P_{2}\left(e^{i t}\right)\right|^{2} d m_{r, y}^{X} \\
& +\rho(\rho-1)\left\{\left\|P_{1}-P_{1}(0)\right\|_{2}^{2}+\left\|P_{2}-P_{2}(0)\right\|_{2}^{2}\|y\|^{2}\right\} \\
& +\frac{\rho-1}{\rho}\left|\left(P_{1}(0)+P_{2}(0)\right)\right|^{2}
\end{aligned}
$$

Proof. Since

$$
J(x)=\int_{0}^{2 \pi}\left[\frac{1-\rho}{\rho}\left(P_{1}(0)+P_{2}(0)\right)+P_{1}\left(e^{-\imath t}\right)+P_{2}\left(e^{\imath t}\right)\right]<K_{r, t}^{\rho}(T) y \mid x>\frac{d t}{2 \pi}
$$

and since $K_{r, t}^{\rho}(T)$ is a positive operator $\left(T \in C_{\rho}\right.$ ), by applying the Cauchy-Schwartz inequality twice, we obtain

$$
\begin{aligned}
|J(x)|^{2} \leq & \int_{0}^{2 \pi}\left|\left(\frac{1-\rho}{\rho}\right)\left(P_{1}(0)+P_{2}(0)\right)+P_{1}\left(e^{-\imath t}\right)+P_{2}\left(e^{\imath t}\right)\right|^{2}<K_{r, t}^{\rho}(T) y \mid y>\frac{d t}{2 \pi} \\
& \times \int_{0}^{2 \pi}<K_{r, t}^{\rho}(T) x \mid x>\frac{d t}{2 \pi} .
\end{aligned}
$$

This leads to the inequality,

$|J(x)|^{2} \leq \rho\|x\|^{2} \int_{0}^{2 \pi}\left|\frac{1-\rho}{\rho}\left(P_{1}+P_{2}\right)(0)+P_{1}\left(e^{-\imath t}\right)+P_{2}\left(e^{i t}\right)\right|^{2}<K_{r, t}^{\rho}(T) y \mid y>\frac{d t}{2 \pi}$. Using the identity $<K_{r, t}^{\infty}(T, X) y\left|y>=\alpha<K_{r, t}(T) y\right| y>$, we get

$$
<K_{r, t}^{\infty}(T, X) y \mid y>=\alpha\left(<K_{r, t}^{\rho}(T) y \mid y>-(\rho-1)\|y\|^{2}\right)
$$

thus

$$
<K_{r, t}^{\rho}(T) y\left|y>=\frac{1}{\alpha}<K_{r, t}^{\infty}(T, X) y\right| y>+(\rho-1)\|y\|^{2} .
$$

Now taking the supermum for $\|x\| \leq 1$ gives

$$
\begin{aligned}
I(y) \leq & \rho \int_{0}^{2 \pi}\left|\frac{1-\rho}{\rho}\left(P_{1}(0)+P_{2}(0)\right)+P_{1}\left(e^{-\imath t}\right)+P_{2}\left(e^{\imath t}\right)\right|^{2}\left\{\frac{1}{\alpha}\left\langle K_{r, t}^{\infty}(T, X) y \mid y\right\rangle\right. \\
& \left.+(\rho-1)\|y\|^{2}\right\} \frac{d t}{2 \pi} \\
= & \frac{\rho}{\alpha} \int_{0}^{2 \pi}\left|\frac{1-\rho}{\rho}\left(P_{1}(0)+P_{2}(0)\right)+P_{1}\left(e^{-\imath t}\right)+P_{2}\left(e^{i t}\right)\right|^{2}\left\langle K_{r, t}^{\infty}(T, X) y\right| y> \\
& +\rho(\rho-1)\|y\|^{2}\left\|\frac{1-\rho}{\rho}\left(P_{1}(0)+P_{2}(0)\right)+P_{1}\left(e^{-\imath t}\right)+P_{2}\left(e^{2 t}\right)\right\|^{2}
\end{aligned}
$$

The last term on the right side of the inequality above equals

$$
\frac{\rho-1}{\rho}\left|\left(P_{1}(0)+P_{2}(0)\right)\right|^{2}+\rho(\rho-1)\left\{\left\|P_{1}-P_{1}(0)\right\|_{2}^{2}+\left\|P_{2}-P_{2}(0)\right\|_{2}^{2}\right\}\|y\|^{2} .
$$

Thus

$$
\begin{aligned}
I(y) \leq & \left.\frac{\rho}{\alpha} \int_{0}^{2 \pi} \mid \frac{1-\rho}{\rho}\left(P_{1}+P_{2}\right)(0)\right)+P_{1}\left(e^{-\imath t}\right)+\left.P_{2}\left(e^{\imath t}\right)\right|^{2} d m_{r, x}^{X}(t) \\
& \left.+\frac{\rho-1}{\rho}\left|\left(P_{1}(0)+P_{2}(0)\right)\right|^{2}+\rho(\rho-1)\left\{\left\|P_{1}-P_{1}(0)\right\|_{2}^{2}+\left\|P_{2}-P_{2}(0)\right\|_{2}^{2}\right]\|y\|^{2}\right\}
\end{aligned}
$$

The proof is complete. 
Remark In the case where $\rho=1$ we obtain for every polynomial $Q$,

$$
\|(Q(T)) y\|\left\|^{2} \leq \frac{1}{\alpha}\right\| \sqrt{X} Q(T) y\left\|^{2} \leq \frac{1}{\alpha}\right\| \sqrt{X}\left\|^{2}\right\| Q(T) y \|^{2}
$$

thus, $T$ is similar to an isometry on $E=\operatorname{span}\left\{T^{n} y, n \geq 0\right\}$. The existence of non trivial invariant subspaces is derived directly from [5].

Proposition 10 Let $T$ be a power bounded operator in the class $C_{\rho}$ and suppose that $\mu_{y, y}^{X}$ is absolutely continuous with respect to the Haar measure $d m=\frac{d t}{2 \pi}$. Define $\Phi$ on polynomials by $\Phi(P)=P(T) y$. Then there exists a positive measure $\nu$ such that $\Phi$ is extendible to $H^{2}(d \nu)$ and intertwines $T$ with the unilateral shift on $H^{2}(d \nu)$

Proof. Before starting the proof, note that every vector, in the singular invariant subspace associated with the singular part of $\mu_{y, y}^{X}$, is non cyclic thus we can assume without loss of generality that $" \mu_{y, y}^{X}$ is absolutely continuous with respect to the Haar measure $d m=\frac{d t}{2 \pi} "$.

For $n \geq 1$, we have $X=T^{* n} X T^{n}$, it follows that

$$
\begin{aligned}
\left.\| T^{n} Q(T)\right) y \|^{2} & \leq \frac{\rho}{\alpha}\left\|\sqrt{X} T^{n} Q(T) y\right\|^{2}+\rho(\rho-1)\left\|Q\left(e^{2 t}\right)\right\|_{2}^{2}\|y\|^{2} \\
& =\frac{\rho}{\alpha}\|\sqrt{X} Q(T) y\|^{2}+\rho(\rho-1)\left\|Q\left(e^{2 t}\right)\right\|_{2}^{2}\|y\|^{2} .
\end{aligned}
$$

If, $Y$ is a canonical Toeplitz, there exists a Banach limit $L$ such that $Y=L\left(T^{* n} T^{n}\right)$. Thus,

$$
\begin{aligned}
\|\sqrt{Y} Q(T) y\|^{2} & =<Y Q(T) y \mid Q(T) y> \\
& \leq \frac{\rho}{\alpha}\|\sqrt{X} Q(T) y\|^{2}+(\rho)(\rho-1)\left[\left\|Q\left(e^{2 t}\right)\right\|_{2}^{2}\|y\|^{2}\right. \\
& =\int_{0}^{2 \pi}\left|Q\left(e^{2 t}\right)\right|^{2} d \nu
\end{aligned}
$$

with $d \nu=\frac{\rho}{\alpha} \mu_{y, y}^{X}+\rho(\rho-1) d m$. Define $\Phi: H^{2}(d \nu) \rightarrow H$ by $\Phi(Q)=Q(T) y$, then $\Phi$ can be extended to $H^{2}(d \nu)$. Let $S_{\nu}$ be the unweighted shift on $H^{2}(d \nu)$, we clearly have,

$$
T \circ \Phi=\Phi \circ S_{\nu}
$$

\section{Cyclic vectors for operators in $C_{R} \cap C_{1,}$.}

Recall that $T \in B(H)$ is said to be a cyclic operator if $E_{T}(x):=\operatorname{span}\left\{T^{n} x,: n \geq 0\right\}=H$ for some $x \in H$ such vector is called a cyclic vector. We denote $C y c(T)$ the set of all cyclic vector for $T$. It is clear that $T$ has a non trivial invariant subspace if and only if $C y c(T) \neq H$. We also see that the lattice of invariant subspaces is large if $C y c(T)=\emptyset$.

Let $R=\sum_{k=0}^{n} a_{k} z^{k}$ be a polynomial and $T$ be an operator in the class $C_{R}$. Since $K_{r, t}^{R}(T) \geq 0$, when $r \rightarrow 1^{-}$, the positive quasi spectral measures $K_{r, t}^{R}(T) d t / 2 \pi$ converge in the weak measure topology to a positive quasi spectral measure $\gamma$. Consequently, the quasi-spectral measure 
$K_{r, t}(T) d t / 2 \pi$ weakly converges to a quasi spectral measure $\mu_{T}$ (not necessarily positive), and we have

$$
\begin{aligned}
\gamma_{x, y}= & \mu_{T, x, y}+\left[\sum_{k=1}^{n} a_{k} e^{-\imath k t}<T^{k} x, y>+\sum_{k=1}^{n} \overline{a_{k}} e^{\imath k t}<T^{* k} x, y>\right. \\
& \left.+\left(\mathcal{R} e\left(a_{0}\right)-1\right)<x, y>\right] \frac{d t}{2 \pi} .
\end{aligned}
$$

for any $(x, y) \in H \times H$. As in the proof of the classical von Neumann inequality given in [2], we can prove a spacial von Neumann inequality associated to the kernel $K_{r, t}^{R}(T)$

$$
\left\|\left[f\left(r T^{*}\right) X+X g(r T)\right] x\right\|^{2} \leq\|X\| \int_{0}^{2 \pi}\left|f\left(e^{-\imath t}\right)+g\left(e^{2 t}\right)\right|^{2}<K_{r, t}^{R}(T, X) x, x>\frac{d t}{2 \pi} .
$$

We investigate in this section the nature of cyclic vectors for operators in $C_{R} \cap C_{1, .}$. We show that if $T$ is an operator in $C_{R} \cap C_{1, .}$, then $T^{N}$ fails to have cyclic vectors for some $N$, and hence the lattice of invariant subspaces of $T^{N}$ is very large. We assume throughout this section that $T$ is injective with dense range. if such assumption is not satisfied the existence of non zero non cyclic vectors is trivial. We first give some preliminary results.

\subsection{Perturbed kernel and absolute continuity.}

The following result is of independent interest and will be used in our proof.

Proposition 11 Let $T$ be an injective operator with dense range and for any positive integer $p$, set $E_{p}=\left\{x, \forall n \geq p \quad T^{* n} T^{n} x=T^{n} T^{* n} x=x\right\}$. Then $E_{p}=E_{0}$ for any $p$, furthermore $T_{\mid E_{0}}$ is a unitary operator and $T_{\mid E_{0}^{\perp}}$ is completely non-unitary.

Proof. Let us first prove that $E$ reduces $T^{p}$ (that is invariant for $T^{p}$ and for $\left(T^{*}\right)^{*}$ ), we claim that if $x \in E$, we have $T^{q} x \in E$ for every $q \geq p$. Indeed

$$
T^{* q}\left(T^{* n} T^{n} T^{q} x-T^{q} x\right)=T^{(n+q) *} T^{n+q} x-T^{* q} T^{q} x=x-x=0,
$$

thus, $T^{* n} T^{n} T^{q} x-T^{q} x \in K e r T^{*}=\overline{R(T)}^{\perp}=\{0\}$ and so, $T^{* n} T^{n} T^{q} x=T^{q} x$ for $n \geq p$. Similarly $T^{n} T^{* n} T^{q} x=T^{q} x$, the claim is proved. $E_{p}$ is invariant for $T^{* q}$ in the same way. For every $x \in E_{p}$ and $y \in E_{p}^{\perp}$, we have

$$
<T x\left|y>=<T T^{p} T^{* p} x\right| y>=<T^{* p} x \mid T^{*(p+1)} y>=0
$$

and since $E_{p}^{\perp}$ is invariant for $T^{p}$ and for $T^{*(p+1)}$, we obtain $E_{p}$ is invariant for $T$. Likewise we see that $E_{p}$ is invariant for $T^{*}$.

To see that $E_{p}=E_{0}$ for $p \geq 0$, let $x$ be in $E_{p}$ and note that

$$
\|T x\|^{2}=\left\|T T^{p} T^{* p} x\right\|^{2}=\left\|T^{p+1}\left(T^{* p} x\right)\right\|^{2}=\left\|T^{* p} x\right\|^{2}=\|x\|^{2} .
$$

Similar arguments show that $\left\|T^{*} x\right\|^{2}=\|x\|^{2}$, for any $x \in E_{p}$. It follows that $\mathcal{T}_{\mid E_{p}}$ is a unitary operator, consequently $E_{p}=E_{0}$. 
To complete the proof, note that if $E$ is a reducing space for $T$ such that $T_{\mid E^{\prime}}$ is a unitary operator, then necessarily $T^{* n} T^{n} x=T^{n} T^{* n} x=x$ and hence $E \subset E_{0}$. In particular $T_{\mid E_{0}^{\perp}}$ is completely non unitary.

\section{Remark.}

1. The set $E_{0}$ considered here was introduced when $T$ is a contraction under the form $\left\{x,\left\|T^{n} x\right\|=\left\|T^{* n} x\right\|=\|x\|\right\},([7]$, Chap 1, theorem 3.2, for example).

2. The proposition holds for some class of operators without the assumption injective with dense range namely normal operators, contractions ...

Proposition 12 Let $T \in C_{R}$ be an injective operator with dense range. If $T$ is completely non unitary, then $T$ is absolutely continuous. Moreover, for every $x \in H \backslash\{0\}$ there exists $h_{x, x} \in L^{1}(d m)$ such that $\ln \left(h_{x, x}\right) \in L^{1}(d m)$ and that satisfies

$$
\gamma_{x, y}=\lim _{w^{*}}<K_{r, t}^{R}(T) x \mid x>d m(t)=h_{x, x} d m .
$$

Proof. For $x, y$ in $H$ we have

$$
<K_{r, t}^{R}(T) x \mid y>d m(t) \rightarrow_{w^{*}} \gamma_{x, y}
$$

and by the Jordan decomposition we get $\gamma_{x, y}=h_{x, y} d m+\nu_{x, y}$ with $h_{x, y} \in L^{1}(d m)$ and $\nu_{x, y}$ a singular measure with respect to the Haar measure. Applying the Cauchy Schwarz inequality to the bilinear application

$$
\begin{array}{ll}
H \text { otimes } H & \rightarrow L^{1}(d m) \\
(x, y) & \mapsto h_{x, y}^{\rho}
\end{array}
$$

gives

$$
\left|\int f\left(e^{\imath t}\right) h_{x, y} d m(t)\right| \leq \sqrt{\int\left|f\left(e^{\imath t}\right)\right| h_{x, x} d m(t)} \sqrt{\int\left|f\left(e^{\imath t}\right)\right| h_{y, y} d m(t)}
$$

for all positive functions. Then it is clear that $F=\left\{x \in H: h_{x, y}=0 \forall y \in H\right\}$ is a closed subspace of $H$. Indeed more is given, $F=\left\{x \in H \backslash h_{x, x}=0\right\}$ and $F^{\perp}=\left\{x \in H: \nu_{x, x}=0\right\}$. Let $\Omega$ be the support of $\nu_{x, y}$, we have $m(\Omega)=0$ since $\nu_{x, y}$ is singular with respect to the Haar measure, there exists a sequence $\left(f_{k}\right)_{k \in \mathbb{N}}$ in the disc algebra $\mathrm{A}(\mathrm{D})$

i) $\left|f_{k}\right| \leq 1$

ii) $f_{k}(z) \rightarrow 1_{\Omega}(z),\left|\nu_{x, y}\right|$-almost everywhere

iii) $f_{k}\left(e^{2 t}\right) \rightarrow 0 m$-almost everywhere (see [11] for more details).

We deduce that $f_{k}(T) \rightarrow \mu_{T}(\Omega)$ in the strong convergence of operators topology with < $\mu_{T}(\Omega)(\varphi) x \mid y>=\int \varphi d \mu_{T, x, y}$. But from equation 4 , we see that

$$
\begin{aligned}
\gamma_{x, y}= & \mu_{T, x, y}+\left[\sum a_{k} e^{-\imath k t}<T^{k} x, y>+\sum \overline{a_{k}} e^{\imath k t}<T^{* k} x, y>\right. \\
& \left.+\left(\mathcal{R} e\left(a_{0}\right)-1\right)<x, y>\right] d m .
\end{aligned}
$$


Thus $\gamma(\Omega)=\mu_{T}(\Omega)=\mu_{T}(\Omega)^{*}$ and $\mu_{T}(\Omega) \in\{T\}^{\prime}$ is an orthogonal projection. In particular $T_{\mid F}$ is unitary. Since $T$ is completely non unitary, we get $F=\{0\}$ and hence $T$ is absolutely continuous.

To prove the second assertion, suppose that $\ln \left(h_{x, x}\right)$ is not integrable. By Szego's formula, we have

$$
\inf _{p \in \mathcal{C}\left[e^{2 \theta}\right]} \int\left|1-e^{\imath \theta} p\left(e^{\imath \theta}\right)\right|^{2} d \gamma_{x, x}=\exp \left[\int \ln \left(h_{x, x}\right) d m\left(e^{\imath \theta}\right)\right]=0 .
$$

We derive that for every $k \geq p=d^{\circ} R+1$, there exists a sequence $\left(p_{n}\right)_{n \in \mathbb{N}} \subseteq \mathrm{C}\left[e^{\imath \theta}\right]$ such that

$$
\int\left|1-e^{\imath(k+p) \theta} p_{n}\left(e^{2 \theta}\right)\right|^{2} d \gamma_{x, x}(\theta) \rightarrow 0
$$

Using spacial Von Neumann's inequality (Equation 4), we get

$$
\left\|T^{p} x-T^{k+2 p} p_{n}(T) x\right\|^{2} \leq \int\left|1-e^{\imath(k+p) \theta} p_{n}\left(e^{\imath \theta}\right)\right|^{2} d \gamma_{x, x}(\theta) \rightarrow 0
$$

and

$$
\left\|T^{* k} x-T^{p} p_{n}(T) x\right\|^{2} \leq \int\left|e^{-\imath k \theta}-e^{\imath p \theta} p_{n}\left(e^{i \theta}\right)\right|^{2} d \gamma_{x, x}(\theta) \rightarrow 0
$$

we deduce that $T^{p} x=\lim _{n \rightarrow+\infty} T^{k+2 p} p_{n}(T) x=T^{k+p} T^{* k} x$ and since $\operatorname{Ker} T=\{0\}$, we obtain $T^{k} T^{* k} x=x$. Using the same arguments, we have $T^{* k} T^{k} x=x$, thus $x \in E_{p}$. Using Proposition 6, we derive that $T_{\mid E_{0}}$ is unitary, this contradicts, $T$ is c.n.u. Finally $\ln \left(h_{x, x}^{\rho}\right)$ is integrable.

Proposition 13 Let $X \in C_{+}(T)$. For every $x, y \in H$ the measure $\mu_{x, y}^{X}$ is absolutely continuous with respect to the Haar measure on the unit circle.

Proof. Let $p$ be a polynomial. For $x, y \in H$, we have

$$
<X p(T) x \mid y>=\int_{0}^{2 \pi} p\left(e^{\imath t}\right) d \mu_{x, y}^{X}(t)
$$

and

If we set

$$
\begin{aligned}
\int_{0}^{2 \pi} p\left(e^{i t}\right) d \gamma_{x, X y}(t) & =\sum_{k=1}^{n} a_{k} \widehat{p}(k)<T^{k} x, X y>+\left(\operatorname{Re}\left(a_{0}\right)-1\right) \\
& <x, X y>+\int_{0}^{2 \pi} p\left(e^{\imath t}\right) d \mu_{T, x, X y}(t) \\
& =\sum_{k=1}^{n} a_{k} \widehat{p}(k)<T^{k} x, X y>+\left(\operatorname{Re}\left(a_{0}\right)-1\right)<x, X y> \\
& +<X P(T) x, y> \\
& =\sum_{k=1}^{n} a_{k} \widehat{p}(k)<T^{k} x, X y>+\left(\mathcal{R} e\left(a_{0}\right)-1\right)<x, X y> \\
& +\int_{0}^{2 \pi} p\left(e^{2 t}\right) d \mu_{x, y}^{X}(t) .
\end{aligned}
$$

$$
\nu=\mu_{x, y}^{X}+\left[\sum_{k=1}^{n} a_{k} e^{\imath k t}<T^{k} x, X y>+\left(\mathcal{R} e\left(a_{0}\right)-1\right)<x, X y>\right] d m-\gamma_{x, y}
$$

we see that $\widehat{\nu}(n)=0$ for $n \geq 0$ then $\nu$ is absolutely continuous with respect to the Haar measure. The preceding lemma asserts that $\gamma_{x, y}$ is absolutely continuous and hence $\mu_{x, y}^{X}$ is absolutely continuous with respect to the Haar measure. 


\subsection{Cyclic vectors}

Now, we give and prove one of the two main theorems of this section.

Theorem 14 Let $T \in C_{R} \cap C_{1, \text {. and }} x \in H$

1. There exists $N_{x} \in \mathbb{N}$ such that $x \notin C y c\left(T^{N}\right)$ and for arbitrary $N \geq N_{x}$.

2. There exists $N_{0} \in \mathbb{N}$ such that $C y c\left(T^{N}\right)=\emptyset$ for any $N \geq N_{0}$.

Proof. 1. Let $x \in H$ be a nonzero element. If $x \notin C y c(T)$, then it is trivial that $x \notin C y c\left(T^{n}\right)$ for every $n \geq 0$. Hence, we can assume that $x \in C y c(T)$. Denote $\mu=\mu_{x, x}^{X}=\varphi d m$ with $\varphi \in L^{1}(d m)$. We have

$$
\|p\|_{L^{2}(\mu)}^{2}=\int_{0}^{2 \pi}\left|p\left(e^{2 t}\right)\right|^{2} d \mu(t)=\|\sqrt{X} p(T) x\|^{2} \leq\|X\|\|p(T) x\|^{2}
$$

and hence the operator $L: p(T) x \in E_{T}(x) \rightarrow p \in H_{\mu}^{2}$ is extendible to a bounded operator on $H$ (that we also denote by $L$ ). The following diagram is commutative.

$$
\begin{array}{lll}
H & \stackrel{L}{\rightarrow} & H_{\mu}^{2} \\
T \downarrow & & \downarrow S_{\mu} \\
H & L & H_{\mu}^{2}
\end{array}
$$

where $S_{\mu}$ is the usual shift on $H_{\mu}^{2}$.

Seeking a contradiction, let $u$ be arbitrary in $H$ and suppose that $u \in E_{T^{n_{k}}}(x)$ for some sequence $n_{k} \geq 0$ that goes to infinity. Thus there exists $p_{n}$ polynomials, such that $u=\lim _{n \rightarrow \infty} p_{n}\left(T^{n_{k}}\right) x$. Using the diagram 4,

$$
L(u)=\lim _{n \rightarrow \infty} L\left(p_{n}\left(T^{n_{k}}\right) x\right)=\lim _{n \rightarrow \infty} p_{n}\left(S_{\mu}^{n_{k}}\right) L x
$$

this leads to the inclusion $\operatorname{Im}(L) \subset \operatorname{span}\left\{\left(S_{\mu}^{n_{k}}\right)^{n} L x: n \geq 0\right\}$ and since $L$ is dense range, we deduce that $\psi:=L(x) \in C y c\left(S_{\mu}^{n_{k}}\right)$ for every $k \geq 0$. We show now that this fact is impossible.

Lemma 15 Let $S_{\mu}$ be the shift operator on $H_{\mu}^{2}$ and $\psi \in H_{\mu}^{2}$. Then there exists $N_{0}$ such that $\psi \notin C y c\left(S_{\mu}^{n_{k}}\right)$ for every $N \geq N_{0}$.

Proof. Suppose that $\psi \notin C y c\left(S_{\mu}^{n_{k}}\right)$ for some given sequence $\left(n_{k}\right)_{k \geq 0}$ tending to infinity. As $\ln (t),(t \in] 0,2 \pi])$ is integrable, there exists $h \in H^{\infty}\left(\subset H_{\mu}^{2}\right)$ such that $\left|h\left(e^{2 t}\right)\right|=t$, a.e with respect to the Lebesgue measure. Thus for some sequence $\left(q_{n}\right)_{n \geq 0}$ of polynomials, we have $\lim _{n \rightarrow \infty}\left\|q_{n} \psi-h \psi\right\|_{H_{\mu}^{2}}=0$. We derive that for $N \geq 0$,

$$
\begin{aligned}
\left.\int_{0}^{2 \pi}|t-| q_{n}\left(e^{i N t}\right)\right|^{2}|\psi|^{2} d m & \leq \int_{0}^{2 \pi}\left|h\left(e^{\imath N t}\right)-q_{n}\left(e^{2 N t}\right) \| \psi\right|^{2} d m \\
& =\left\|q_{n} \psi-h \psi\right\|_{H_{\mu}^{2}} \rightarrow 0 \text { when } n \rightarrow \infty
\end{aligned}
$$


Let $\Omega$ be the support of $\psi$, there exists a subsequence of $q_{n}$ that we denote $q_{n_{k}}$ such that

$$
q_{n_{k}}\left(e^{i N t}\right) \rightarrow t \text { a.e with respect to Lebesgue measure. }
$$

If we set $\Omega_{k}=\Omega-\frac{2 k \pi}{N}$ for $0 \leq k \leq N-1$, then $\Omega_{k} \cap \Omega_{p}=\emptyset$ for $0 \leq k, p \leq N-1$. To see this consider $t \in \Omega_{k} \cap \Omega_{p}$, and write $t=t_{1}+\frac{2 k \pi}{N}=t_{2}+\frac{2 p \pi}{N}$. Then by equation 5 since $0 \leq p, k \leq N-1$, we obtain $t_{1}=t_{2}$ and $p=k$. To get our contradiction, note that

$$
\begin{aligned}
1 & =m(T) \geq \sum_{k=0}^{N-1} m\left(\Omega_{k}\right) \\
& =N m(\Omega) \quad \text { (Invariance by translations) }
\end{aligned}
$$

for every $N$, hence $m(\Omega)=0$. Which is impossible.

2. In a similar way 2. is a direct consequence of the following lemma.

Lemma 16 Let $S_{\mu}$ be the shift operator on $H_{\mu}^{2}$ and $\Omega=\operatorname{supp}(\mu)$. Then for every $N>\frac{1}{m(\Omega)}$, the operator $S_{\mu}^{N}$ has no cyclic vector.

Proof. It outlines the proof of Lemma 15 by observing that $\Omega \subset \operatorname{supp}(\phi)$ for every cyclic vector for $S_{\mu}^{n}$.

Remark. Theorem 14 gives a rich set of invariant subspaces for some power of $T$ but does not seem to give any information about invariant subspaces for $T$ that arise directly from non cyclic vectors. If $S$ is the usual shift on the Hardy space $H^{2}$, then an easy proof shows that $S^{2}$ has no cyclic vectors (a fact that is also a consequence of Lemma 16) while $S$ has a large set of cyclic vectors. Namely all outer functions.

In [7] B. Nagy and C. Foias were interested in the following problem. Let $T$ be a contraction in the class $C_{1, .}$,

under which assumption, $T$ is cyclic implies $T^{*}$ is cyclic?

The rest of this section deals with this problem for power bounded operators in the class $C_{1, .}$. We generalize their result to such operators by relying generalized Toeplitz operators, cyclic vectors for $T$ and cyclic vectors for $T^{*}$.

Theorem 17 Let $T$ be a power bounded operator of class $C_{1, .}, X \in C_{+}(T)$ be injective.

1. If $D \cap\left(\sigma(T) \backslash \sigma_{p}(T)\right) \neq \emptyset$, then $X(C y c(T)) \subset C y c\left(T^{*}\right)$. In particular $T^{*}$ is cyclic.

Moreover, if $T$ is completely non unitary the assumption $D \cap\left(\sigma(T) \backslash \sigma_{p}(T)\right) \neq \emptyset$ is no longer needed to say that $T^{*}$ is cyclic and we have

2 If $\ln \left(\mu_{a, a}^{X} / d m\right)$ is not a Lebesgue integrable function, then $X\left(C y c(T) \subset C y c\left(T^{*}\right)\right.$.

3 If the function $\ln \left(\mu_{a, a}^{X} / d m\right)$ is Lebesgue integrable, then $\operatorname{Im}\left(\sqrt{X} \cap C y c\left(T^{*}\right) \neq \emptyset\right.$ 
Proof. Fix $a \in C y c(T)$ and write $\sqrt{X} T=U \sqrt{X}$ and suppose that $T^{*}-\lambda$ is injective for some $\lambda \in \sigma(T)$ such that $|\lambda|<1$. From the equality $\left(T^{*}-\lambda\right) \sqrt{X}=\sqrt{X}\left(U^{*}-\lambda\right)$, it follows that $\lambda \notin \sigma_{p}\left(U^{*}\right)$. In particular $U-\bar{\lambda}$ has a dense range, and since it is closed range we get $U-\bar{\lambda}$ is onto. We conclude that, because $U$ is an isometry, $U$ is a unitary transformation. On the other hand, observing that $\sqrt{X} P(T) a=P(U) \sqrt{X} a$ and using the fact that $X$ has a dense range, we have $b=X a$ is a cyclic vector for $U$. But for unitary transformation (in fact for normals) we have $\left\|q(U) b-\bar{q}\left(U^{*}\right) b\right\|=\left\|\bar{q}\left(U^{*}\right) b-q(U) b\right\|$ for all polynomials $P, Q$. This leads to $\sqrt{X} a$ is a cyclic vector for $U^{*}$ and it follows that $X a$ is a cyclic vector for $T^{*}$.

By Proposition 11, we know that $\mu_{a, a}^{X}$ is absolutely continuous with respect to $m$, thus there exists $\varphi \in L^{1}(d m)$ such that $\mu_{a, a}^{X}=\varphi d m$. We have the following alternative:

$\left.1^{\circ}\right)$ The function $\ln h_{a, a}=\ln \varphi$ is not a Lebesgue integrable. Using Szego's theorem, we see that for every positive integer $p$ there exists a sequence $\left(p_{n}\right)_{n \geq 0} \subseteq \mathrm{C}\left[e^{\imath \theta}\right]$ such that $\int \mid 1-$ $\left.e^{\imath(p+1) \theta} p_{n}\left(e^{\imath \theta}\right)\right|^{2} \varphi\left(e^{\imath \theta}\right) d m(\theta) \rightarrow 0$. If $p(z)=\sum_{k=1}^{n} \widehat{p}(k) z^{k}$ is a polynomial, we denote by $\bar{p}$ the polynomial given by $\bar{p}(z)=\sum_{k=1}^{n} \overline{\widehat{p}(k)} z^{k}$. By proposition 6 , we have

$$
\left\|\bar{p}\left(T^{*}\right) T^{*} X a-X T^{p} a\right\|^{2} \leq\|X\|^{2} \int_{0}^{2 \pi}\left|\overline{p\left(e^{2 t}\right)} e^{-\imath t}-e^{\imath p t}\right|^{2} \varphi\left(e^{\imath t}\right) d m(t) \rightarrow 0
$$

Let us denote by $F=V_{k \geq 0} T^{* k}(X a)$ the closed subspace generated by the sequence $\left(T^{* k}(X a)\right)_{k \geq 0}$. The previous inequality implies that $T^{p}(a) \in F$ for any $p \geq 0$. Therefore, we get that

$$
X(H)=X\left(\bigvee_{k \geq 0} T^{k}(a)\right) \subseteq F
$$

Since $X$ is an injective positive operator, we have $H=\overline{X(H)} \subseteq F$, thus $F=H$ and $X a$ is cyclic for $T^{*}$.

$\left.2^{\circ}\right)$ The function $\ln h_{a, a}=\ln \varphi$ is a Lebesgue integrable. Then, there exists $\psi$ in $H^{2}$ such that $\varphi\left(e^{\imath t}\right)=\left|\psi\left(e^{\imath t}\right)\right|^{2}$. Since $X$ is an injective operator which belongs to $C_{+}(H)$, by the polar decomposition there exists an isometry $V$ such that $\sqrt{X} T=V \sqrt{X}$. Observe that

$$
\|p(V) \sqrt{X} a\|^{2}=\int_{0}^{2 \pi}\left|p\left(e^{\imath t}\right)\right|^{2}\left|\psi\left(e^{2 t}\right)\right|^{2} d m(t)
$$

and it follows that $b=\sqrt{X} a$ is cyclic for $V$. On the other hand, the previous formula shows that the operator $W: p(V) b \in H \rightarrow p\left(e^{2 t}\right) \in H_{\mu}^{2}$ is extendible to a unitary operator (that we also denote $W$ ). Let $S_{\gamma}$ be the usual shift on the space $H^{2}(\gamma)$, where $\gamma$ is the measure given by $\gamma=\left|\psi\left(e^{\imath t}\right)\right|^{2} d m(t)$. Since we clearly have $W \circ V=S_{\gamma} \circ W$, we get that $V$ is unitarily similar to $S_{\gamma}$. The operator $S_{\gamma}^{*}$ clearly admits cyclic vectors (for instance, every vector $\phi / \psi$ where $\phi$ is a cyclic vector for the usual backward shift). It follows immediately that $V^{*}$ admits cyclic vectors, let $b_{0}$ be such cyclic vector. Since $X$ is a positive operator with a dense range, the relation $T^{*} \sqrt{X}=\sqrt{X} V^{*}$ ensures that $b=\sqrt{X} b_{0}$ is cyclic for $T^{*}$. This ends the proof.

As a corollary we retrieve the first result in [7]. 
Corollary 18 Let $T$ be a cyclic contraction in the class $C_{1, .}$. Then $T^{*}$ is cyclic in each of the following cases

1. If $D \cap\left(\sigma(T) \backslash \sigma_{p}(T)\right) \neq \emptyset$.

2. $T$ is a completely non unitary.

\subsection{Cyclic vectors and the commutativity of the commutant}

The second problem treated in [7] is the following. Let $T$ be a contraction in the class $C_{1, .}$. Under which conditions is the commutant $\{T\}^{\prime}$ the commutant of $T$ is commutative?

In this section, we describe the commutant of a power bounded operator and deduce its commutativity under more general assumptions.

We first give a generalization of a theorem in [5]. The proof runs in a similar way and will be omitted.

Theorem 19 Let $T \in P W B(H)$ and $X \in \tau(T)$. Assume that $T$ is cyclic. Then the map

$$
f \rightarrow \mu^{X}(f)
$$

is a linear surjection from $L^{\infty}\left(\mu^{X}\right)$ onto $C(T)$, which induces a faithful order preserving surjection from $L^{\infty}\left(\mu^{X}\right)_{+}$onto $C_{+}(T)$.

We have

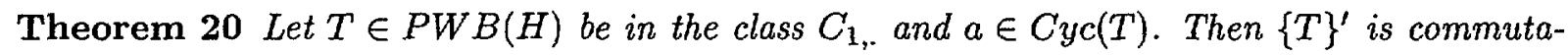
tive. Moreover, for any $X \in \tau(T)$, we have

$$
\overline{X\{T\}^{\prime}}=\mu^{X}\left(H^{\infty}\left(\mu_{a, a}^{X}\right)\right)
$$

where $H^{\infty}\left(\mu_{a, a}^{X}\right)$ is the weak closure of polynomials is $L^{\infty}\left(\mu_{a, a}^{X}\right)$.

Proof. The proof lies in the two following ingredients.

Ingredient 1. For any $R \in\{T\}^{\prime}$ and $p_{n}$ such that $p_{n}(T) a \rightarrow R a$, the sequence $p_{n}$ converges in $L^{2}\left(\mu^{X}\right)$ to the symbol associated with $X R=\mu^{X}(\phi)$ by the previous theorem. Indeed, since

$$
\left\|X p_{n}(T) a-X p_{m}(T) a\right\|^{2}=\int_{0}^{2 \pi}\left|p_{n}\left(e^{i t}\right)-p_{m}\left(e^{\imath t}\right)\right|^{2} d \mu_{a, a}^{X}
$$

the sequence $p_{n}$ converges to a function $\psi \in L^{2}\left(\mu^{X}\right)$. Observe that for any polynomial $p, q$

$$
\begin{aligned}
\int_{0}^{2 \pi} \phi\left(e^{\imath t}\right) p\left(e^{\imath t}\right) \bar{q}\left(e^{\imath t}\right) d \mu_{a, a}^{X} & =<\mu^{X}(\phi) p(T) a, q(T) a> \\
& =<X R p(T) a, q(T) a> \\
& =\lim _{n \rightarrow+\infty}<X p_{n}(T) p(T) a, q(T) a> \\
& =\lim _{n \rightarrow+\infty} \int_{0}^{2 \pi} \phi\left(e^{\imath t}\right) p_{n}\left(e^{\imath t}\right) p\left(e^{i t}\right) \overline{q\left(e^{2 t}\right)} d \mu_{a, a}^{X} \\
& =\int_{0}^{2 \pi} \psi\left(e^{\imath t}\right) p\left(e^{\imath t}\right) \overline{q\left(e^{2 t}\right)} d \mu_{a, a}^{X}
\end{aligned}
$$


Hence $\psi=\phi d \mu_{a, a}^{X}$ a.e and since $a \in c y c(T)$, it follows that $\psi=\phi d \mu^{X}$ a.e.

Ingredient 2. Let $R_{1}$ and $R_{2}$ be in the commutant of $T$ and write $R_{1} a=\lim _{n \rightarrow+\infty} p_{n}(a)$ and $R_{2} a=\lim _{n \rightarrow+\infty} q_{n}(a)$. Fix $f_{1}=\lim p_{n}$ and $f_{2}=\lim q_{n}$ obtained by Ingredient 1.. Since $X_{1}=X R_{1}$ and $X_{2}=X R_{2}$ belong to $C(T)$, using again the previous theorem, there exists $f_{1}$, and $f_{2}$ in $H^{\infty}\left(\mu^{X}\right)$ such that $X_{1}=\mu^{X}\left(f_{1}\right)$ and $X_{2}=\mu^{X}\left(f_{2}\right)$. For any $r, s$ polynomials, we have

$$
\begin{aligned}
\left\langle X_{1} R_{2} r(T) a, s(T) a>\right. & =\lim _{n \rightarrow+\infty}<X q_{n}(T) r(T) a, s(T) a> \\
& =\lim _{n \rightarrow+\infty} \int_{0}^{2 \pi} q_{n}\left(e^{2 t}\right) r\left(e^{\imath t}\right) \overline{s\left(e^{2 t}\right)} f_{1}\left(e^{2 t}\right) d \mu_{a, a}^{X} \\
& =\int_{0}^{2 \pi} f_{2}\left(e^{\imath t}\right) r\left(e^{2 t}\right) \overline{s\left(e^{2 t}\right)} f_{1}\left(e^{\imath t}\right) d \mu_{a, a}^{X}
\end{aligned}
$$

Similarly, $\left\langle X_{2} R_{1} r(T) a, s(T) a\right\rangle=\int_{0}^{2 \pi} f_{2}\left(e^{\imath t}\right) r\left(e^{\imath t}\right) \bar{s}\left(e^{\imath t}\right) f_{1}\left(e^{\imath t}\right) d \mu_{a, a}^{X}$.

Thus $X R_{1} R_{2}=X_{2} R_{1}=X_{1} R_{2}=X R_{2} R_{1}$. The injectivity of $X$ leads to $R_{1} R_{2}=R_{2} R_{1}$.

The commutativity of $\{T\}^{\prime}$ is obtained.

From the proof of the first assumption, we see that $\overline{X\{T\}^{\prime}} \subset H^{\infty}\left(\mu^{X}\right)$. To show the reverse inclusion, it suffices to use again Theorem 19.

\section{The perturbed kernel and spacial von Neumann inequalities.}

The aim of this section is to provide asymptotic Von Neumann inequality associated with perturbed kernels. We give complete computation in the case where the perturbation is $R(T)=$ $\bar{\beta} T+\beta T^{*}+I$. The general case of trigonometric perturbation follows immediately.

Let $T \in L(H)$ and $\beta \in C$. We denote the perturbed kernel associated with $T$,

$L_{\beta}(T)=K_{\beta}(T)+\bar{\beta} T+\beta T^{*}$, the asymptotic perturbed kernel associated with $T, L_{\beta}(T, X)=$ $L_{\beta}(T, X)+\bar{\beta} X T+\beta T^{*} X$, and the $\rho$-perturbed kernel associated with $T L_{\beta}^{\rho}(T)=K_{\beta}^{\rho}(T)+\bar{\beta} T+$ $\beta T^{*}$. Then, direct computations give

1. $\left\langle L_{\beta}(T, X) y\left|y>=\alpha<L_{\beta}(T) y\right| y>\right.$.

2. $f(r T)=\int_{0}^{2 \pi} f\left(e^{\imath t}\right) L_{\beta}(T)(T) \frac{d t}{2 \pi}-|\beta| f^{\prime}(0) T$.

3. $f\left(r T^{*}\right)=\int_{0}^{2 \pi} f\left(e^{-\imath t}\right) L_{\beta}(T)(T) \frac{d t}{2 \pi}-|\beta| f^{\prime}(0) T^{*}$.

We set

$$
J(x)=<\left(P_{1}\left(r T^{*}\right)+P_{2}(r T)\right) y \mid x>.
$$

Then

$$
\begin{aligned}
J(x)= & \int_{0}^{2 \pi}\left[\left(\frac{1-\rho}{\rho}\right)\left(P_{1}(0)+P_{2}(0)\right)+\left(P_{1}\left(e^{-\imath t}\right)+P_{2}\left(e^{\imath t}\right)\right]<K_{r, t}^{\rho}(T) y|x\rangle \frac{d t}{2 \pi}\right. \\
= & \int_{0}^{2 \pi}\left[\left(\frac{1-\rho}{\rho}\right)\left(P_{1}(0)+P_{2}(0)\right)+\left(P_{1}\left(e^{-i t}\right)+P_{2}\left(e^{\imath t}\right)\right]<L_{\beta}^{\rho}(T) y|x\rangle\right. \\
& -<\bar{\beta} T+\beta T^{*} y \mid x>\frac{d t}{2 \pi} \\
= & \int_{0}^{2 \pi}\left[\left(\frac{1-\rho}{\rho}\right)\left(P_{1}(0)+P_{2}(0)\right)+\left(P_{1}\left(e^{-\imath t}\right)+P_{2}\left(e^{\imath t}\right)\right]\right. \\
& \cdot<L_{\beta}^{\rho}(T) y\left|x>\frac{d t}{2 \pi}-\right| \beta \mid\left(P_{1}^{\prime}(0)<T y|x>-| \beta\left|P_{2}^{\prime}(0)<T^{*} y\right| x>\right.
\end{aligned}
$$


By the Cauchy-Schwartz inequality

$$
\begin{aligned}
|J(x)| \leq & \left(\int _ { 0 } ^ { 2 \pi } \left[\mid\left(\frac{1-\rho}{\rho}\right)\left(P_{1}(0)+P_{2}(0)\right)+\left(P_{1}\left(e^{-\imath t}\right)+\left.P_{2}\left(e^{\imath t}\right)\right|^{2}\right]\right.\right. \\
& \left.\cdot<L_{r, t}^{\rho}(T) y\left|y>\frac{d t}{2 \pi} \int_{0}^{2 \pi}<L_{r, t}^{\rho}(T) x\right| x>\frac{d t}{2 \pi}\right)^{\frac{1}{2}} \\
& +\mid \beta \|\left(P_{1}^{\prime}(0)<T y|x>+| \beta\left|P_{2}^{\prime}(0)<T^{*} y\right| x>\mid\right.
\end{aligned}
$$

we obtain

$$
\begin{aligned}
|J(x)| \leq & \rho^{\frac{1}{2}}\|x\| \int_{0}^{2 \pi}\left[\mid\left(\frac{1-\rho}{\rho}\right)\left(P_{1}(0)+P_{2}(0)\right)+\left(P_{1}\left(e^{-\imath t}\right)+\left.P_{2}\left(e^{\imath t}\right)\right|^{2}\right]\right. \\
& \cdot<L_{\beta}^{\rho}(T) y \mid y>\frac{d t}{2 \pi} \\
& +\mid \beta \|\left(P_{1}^{\prime}(0)\|T y\|+|\beta| P_{2}^{\prime}(0)\left\|T^{*} y\right\|\|x\| .\right.
\end{aligned}
$$

As $\left\langle L_{\beta}^{\rho}(T) y\left|y>=\frac{1}{\alpha}<L_{\beta}(T, X) y\right| y>+(\rho-1)\|y\|^{2}\right.$, by the preceding inequality and taking the supermum for $\|x\| \leq 1$, we get

$$
\begin{aligned}
|J(y)| \leq & \frac{\rho}{\alpha} \frac{1}{2}\left[\int_{0}^{2 \pi} \mid\left(\frac{1-\rho}{\rho}\right)\left(P_{1}(0)+P_{2}(0)\right)\right. \\
& +\left(P_{1}\left(e^{-2 t}\right)+\left.P_{2}\left(e^{2 t}\right)\right|^{2}<L_{\beta}(T, X) y \mid y>\frac{d t}{2 \pi}\right. \\
& \left.\left.+\frac{\rho}{(\rho-1)}\left[\left\|P_{1}+P_{2}\right\|_{2}^{2}+\left(\frac{1-\rho^{2}}{\rho^{2}}\right)\left|\left(P_{1}(0)+P_{2}(0)\right)\right|^{2}\right]\|y\|^{2}\right)\right]^{\frac{1}{2}} \\
& +\mid \beta \|\left(P_{1}^{\prime}(0)\|T y\|+P_{2}^{\prime}(0)\left\|T^{*} y\right\| .\right.
\end{aligned}
$$

Remarks It is easy to see that if we take, $R(T)=\bar{\beta}^{n} T^{n}+\beta^{n} T^{n *}$, then

$$
\int_{0}^{2 \pi} f\left(e^{i t}\right) K_{\beta}(T)(T) \frac{d t}{2 \pi}=\int_{0}^{2 \pi} f\left(e^{2 t}\right) L_{\beta}(T)(T) \frac{d t}{2 \pi}-|\beta| f^{(n)}(0) T
$$

the corresponding von Neumann asymptotic inequality is

$$
\begin{aligned}
|J(y)| \leq & \frac{\rho}{\alpha} \frac{1}{2}\left[\int_{0}^{2 \pi} \mid\left(\frac{1-\rho}{\rho}\right)\left(P_{1}(0)+P_{2}(0)\right)+\left(P_{1}\left(e^{-\imath t}\right)\right.\right. \\
& \left.+\left.P_{2}\left(e^{2 t}\right)\right|^{2} \cdot<L_{r e^{2 t}}(T, X) y \mid y>\frac{d t}{2 \pi}\right] \\
& \left.\left.+\frac{\rho}{(\rho-1)}\left[\left\|P_{1}+P_{2}\right\|_{2}^{2}+\left(\frac{1-\rho^{2}}{\rho^{2}}\right)\left|\left(P_{1}(0)+P_{2}(0)\right)\right|^{2}\right]\|y\|^{2}\right)\right]^{\frac{1}{2}} \\
& +|\beta|^{n} P_{1}^{(n)}(0)\left\|T^{n} y\right\|+|\beta|^{n} \mid P_{2}^{(n)}(0)\left\|T^{* n} y\right\| .
\end{aligned}
$$

If we consider $L_{r e^{2 t}}(T)=K_{r e^{2 t}}(T)+\sum_{\jmath=1}^{\jmath=n} \nu_{\jmath} e^{\imath \jmath t} T^{\jmath}+\sum_{\imath=1}^{j=n} \mu_{\jmath} e^{-\imath \jmath t} T^{* j}$ we get by linearity,

$$
\begin{aligned}
|J(y)| \leq & \frac{\rho}{\alpha} \frac{1}{2}\left[\int_{0}^{2 \pi} \mid\left(\frac{1-\rho}{\rho}\right)\left(P_{1}(0)+P_{2}(0)\right)+\left(P_{1}\left(e^{-\imath t}\right)\right.\right. \\
& +\left.P_{2}\left(e^{2 t}\right)\right|^{2} \cdot<L_{r e^{2 t}}(T, X) y \mid y>\frac{d t}{2 \pi} \\
& \left.\left.+\frac{\rho}{(\rho-1)}\left[\left\|P_{1}+P_{2}\right\|_{2}^{2}+\left(\frac{1-\rho^{2}}{\rho^{2}}\right)\left|\left(P_{1}(0)+P_{2}(0)\right)\right|^{2}\right]\|y\|^{2}\right)\right]^{\frac{1}{2}} \\
& +\sum_{j=1}^{j=i n f\left(n, \operatorname{deg} P_{1}\right)}\left|\nu_{\jmath}\right| P_{1}^{(j)}(0)\left\|T^{\jmath} y\right\| \\
& +\sum_{i=1}^{j=2 n f\left(n, \operatorname{deg} P_{2}\right)}\left|\mu_{\jmath}\right| P_{2}^{(\jmath)}(0)\left\|T^{* j} y\right\| .
\end{aligned}
$$

Acknowledgments. The authors thank the Abdus Salam ICTP for the kind support provided during the preparation of part of this paper. The two last authors acknowledge the Mathematics Department of University Lyon 1, where this work was initiated. 


\section{References}

[1] B. Beauzamy, Sous-espaces de type fonctionnel dans les espaces de Banach. Acta. Math. $144(1980) 65-82$.

[2] G. Cassier, Ensemble $K$ spectraux et algèbres duales d'opérateurs, Preprint LAFP,

[3] G. Cassier, Autour de quelques interactions récentes entre l'analyse complexe et la théorie des opérateurs. Actes du colloque international de Rabat (1999) Theta Foundation, to appear.

[4] G. Cassier, Generalized Toeplitz operators, restriction to invariant subspaces and similarity problems. Preprint

[5] G. Cassier and T. Fack, Contractions in Von Neumann Algebras. J. Funct. Anal. $135(2)(1996) 297-338$.

[6] G. Eckstein, Sur les opérateurs de classe $C_{\rho}$. Acta.Sci.Math 33(1972)354-352.

[7] B. Sz Nagy and C.Foias, Harmonic analysis of operators on Hilbert spaces. North holland. Amsterdam. 1970.

[8] B. Sz Nagy and C.Foias, Contractions without cyclic vectors, Proc. Amer. Math. Soc. $87(4)(1983) 671-674$.

[9] B. Sz Nagy and C.Foias, Vecteurs cycliques et commutativité du commutant. Acta Sci. Math., (1970) 177-183.

[10] B. Sz Nagy and C.Foias, Harmonic analysis of operators on Hilbert spaces. North holland. Amsterdam. 1970.

[11] K. Hoffman, Banach spaces of analytic functions. Reprint of the 1962 original. Dover Publications, Inc., New York, 1988. 